\title{
A Scalable and Efficient Approach for Compiling and Analyzing Commit History
}

\author{
Pooyan Behnamghader \\ University of Southern Califronia \\ pbehnamg@usc.edu \\ Di Huang \\ University of Southern Califronia \\ huangdi@usc.edu
}

\author{
Patavee Meemeng \\ University of Southern Califronia \\ meemeng@usc.edu \\ Kamonphop Srisopha \\ University of Southern Califronia \\ srisopha@usc.edu
}

\author{
Iordanis Fostiropoulos \\ University of Southern Califronia \\ fostirop@usc.edu \\ Barry Boehm \\ University of Southern Califronia \\ boehm@usc.edu
}

\begin{abstract}
Background: Researchers oftentimes measure quality metrics only in the changed files when analyzing software evolution over commithistory. This approach is not suitable for compilation and using program analysis techniques that require byte-code. At the same time, compiling the whole software not only is costly but may also leave us with many uncompilable and unanalyzed revisions. Aims: We intend to demonstrate if analyzing changes in a module results in achieving a high compilation ratio and a better understanding of software quality evolution. Method: We conduct a large-scale multiperspective empirical study on 37838 distinct revisions of the core module of 68 systems across Apache, Google, and Netflix to assess their compilability and identify when the software is uncompilable as a result of a developer's fault. We study the characteristics of uncompilable revisions and analyze compilable ones to understand the impact of developers on software quality. Results: We achieve high compilation ratios: $98.4 \%$ for Apache, $99.0 \%$ for Google, and 94.3\% for Netflix. We identify 303 sequences of uncompile commits and create a model to predict uncompilability based on commit metadata with an F1-score of 0.89 and an AUC of 0.96 . We identify statistical differences between the impact of affiliated and external developers of organizations. Conclusions: Focusing on a module results in a more complete and accurate software evolution analysis, reduces the cost and complexity, and facilitates manual inspection.
\end{abstract}

\section{CCS CONCEPTS}

- Software and its engineering $\rightarrow$ Software configuration management and version control systems; Maintaining software;

\section{KEYWORDS}

Software maintainbaility evolution; mining software repositories; software compilability; software technical debt;

\footnotetext{
Permission to make digital or hard copies of all or part of this work for personal or classroom use is granted without fee provided that copies are not made or distributed for profit or commercial advantage and that copies bear this notice and the full citation on the first page. Copyrights for components of this work owned by others than the author(s) must be honored. Abstracting with credit is permitted. To copy otherwise, or republish, to post on servers or to redistribute to lists, requires prior specific permission and/or a fee. Request permissions from permissions@acm.org.

ESEM '18, October 11-12, 2018, Oulu, Finland

(C) 2018 Copyright held by the owner/author(s). Publication rights licensed to the Association for Computing Machinery.

ACM ISBN 978-1-4503-5823-1/18/10 ..\$15.00

https://doi.org/10.1145/3239235.3239237
}

ACM Reference Format:

Pooyan Behnamghader, Patavee Meemeng, Iordanis Fostiropoulos, Di Huang, Kamonphop Srisopha, and Barry Boehm. 2018. A Scalable and Efficient Approach for Compiling and Analyzing Commit History. In ACM / IEEE International Symposium on Empirical Software Engineering and Measurement (ESEM) (ESEM '18), October 11-12, 2018, Oulu, Finland. ACM, New York, NY, USA, 10 pages. https://doi.org/10.1145/3239235.3239237

\section{INTRODUCTION}

A software system may undergo thousands of changes over its development history. These changes are recorded by commits in modern version control systems. A commit contains a wealth of information on when, why, and by whom software has changed.

Due to the cost and complexity involved in large-scale software evolution analysis by commit-level, researchers oftentimes achieve scale and omit redundancy by running static analysis on distinct revisions of each file $[1,7,19,22]$. This approach is extremely efficient in generating an Abstract Syntax Tree and calculating filebased quality metrics. Nevertheless, separately analyzing distinct revisions of each file is not suitable when the analysis requires compilation and/or considers relationships between files.

At the same time, compiling the whole software after every commit not only is costly but may also leave us with many uncompilable revisions since only a one-line syntax error is enough to break the build for the whole software. A recent study [20] that mines the commit history of 100 Apache systems indicates that only $4 \%$ of systems exhibit no compile error, $62 \%$ of all commits are currently not compilable, and $58 \%$ of errors are related to resolution of artifacts.

Some static and dynamic program analysis techniques (e.g., architecture recovery [12] and test-coverage [14]) depend on the availability of byte-code and cannot analyze uncompilable revisions. Consequently, utilizing them to measure software quality after each commit results in an incomplete analysis since multiple revisions are left unanalyzed. Alexandru et al. [1] declare the unavailability of compiled versions to be the main unresolved source for the manual effort in software evolution analysis.

In this paper, we take steps to achieve a high compilation ratio by focusing on an evolving module instead of the changed files or the whole software when analyzing commit history. We compile and analyze only the distinct revisions of that module and omit other modules to prevent their errors from breaking the compilation and causing missing of data. We reach the maximum compilation over commit history for that module and identify all commits that are uncompilable as a result of a developer's fault during development. 
To demonstrate the efficiency of our approach, we conduct a large-scale empirical study on 37838 distinct revisions of the core module of 68 open-source software systems across Apache, Google, and Netflix to answer four research questions: 1) How efficient is our approach in reaching the maximum compilation and identifying uncompilable commits? 2) What are the characteristics of sequences of uncompilable commits? 3) Is it feasible to predict uncompilability based on commit metadata? 4) How efficient is our approach in identifying change in quality metrics?

Based on our empirical evaluation, we discuss how focusing on an evolving module achieves a more complete and accurate analysis of software quality evolution, facilitates manual inspection of data, and reduces the cost and complexity of analysis. We detail our algorithms to identify ancestry relationships between distinct revisions of a module, as well as the architecture of SQUAAD, our automated cloud-based framework that facilitates and scales compilation and analysis of source/byte code over commit history.

The remainder of the paper is organized as follows. Section 2 explains related work. Section 3 details how to identify distinct revisions of a module and ancestry relationships between them, how to reach the maximum compilation, and how to scale the analysis. Section 4 explains an empirical evaluation of our approach. Section 5 discusses benefits and risks of focusing on a module based on our empirical analysis and threats to validity of our study. Section 6 concludes the paper.

\section{RELATED WORK}

We focus the related work discussion in two areas: compilability $[9,10,13,15,16,20]$ and software quality evolution $[1,7,17-19,22]$ by commit-level.

Compilability. Multiple recent publications have assessed the compilability of software repositories. Some studies $[9,16]$ analyze compilability only in the last commit in the repository. Some $[10,15]$ analyze the log files recorded by continuous integration frameworks. The studies that compile commit history either focus on missing dependencies [13] or achieve a low compilation ratio [20].

Hassan et al. [9] analyze the possibility of automatically building the last commit for the top 200 Java repositories on Github. They use default Ant, Maven, and Gradle commands to automatically build the systems. For those that fail, they manually fix the compile errors and find that at least $57 \%$ of build failures can be automatically resolved. Sulír et al. [16] automatically build the most recent version of 7200 open-source Java systems and analyze failures. They conclude that the most frequent errors are related to dependencies, Java compilation, and documentation generation.

Seo et al. [15] analyzes 26.6 million builds produced during a period of 9 months by Google engineers, focusing on the frequency and the cause of build failure as well as how long it takes to fix the issues. Their work is focused on developer's build activity rather than commit history. Hassan et al. [10] propose a build-outcome prediction model based on combined features of build-instance metadata and code difference information of commit to predict whether a build will be successful without attempting actual build. They use a dataset recorded by continuous integration practices containing more than 250,000 build instances over a period of 5 years for training and achieve the outcome with an average $\mathrm{F}$ Measure over $87 \%$.
Macho et al. [13] identify 125 commits in 23 repositories that repair a missing dependency, qualitatively and quantitatively analyze how the fix is applied, and propose an approach to automatically fix dependency build breakage. Tufano et al. [20] study the compilability of 219,395 snapshots of 100 Java projects from Apache Foundation. They analyze how frequent broken snapshots happen and the major possible causes behind them. They indicate that surprisingly only $38 \%$ of the commit history is currently compilable. They found that the snapshots are broken mainly due to problems of resolution of artifacts. In our previous work [3], we compile the commit history of the core module of 38 Apache systems and achieve a $98 \%$ compilation ratio on average. We provide guidelines to prevent developers from accidentally committing broken code, but we do not quantify characteristics of uncompilable commits.

Software Quality Evolution. Over the past few years, multiple automated tools have been developed to collect quality metrics over the full commit history. However, none is capable of compilation.

Boa $[7,17]$ is a query system developed specifically for mining repositories. The original version of Boa indexes metadata information about projects, authors, revision history and files and stores no source-code facts. The newer version parses each Java file to extract and store the Abstract Syntax Tree. Lisa [1] is a tool for reducing redundancies in multi-revision code analysis. It runs the analysis on every file in the bare local Git repository at the start of the analysis. It saves the artifacts, then loads the already analyzed results for each file when analyzing every revision, instead of checking out all files of that revision and running the analysis technique. This reduces the amortized time of the analysis for each revision.

HistoryMiner [22] is a tool to conduct large-scale empirical study on when and why code starts to smell bad and when it disappears. It mines every commit in the history of software and applies a lightweight static analysis technique on files affected by the commit to check if a code smell is introduced. SmartShark $[18,19]$ is a distributed framework designed to address the problems with external validity of mining software repository studies. It runs static analysis on affected files by every commit. It constructs the AST and calculates metrics such as number of clone for each file. Its distribution approach is designed to perform on HPC clusters.

In summary, there is no approach capable of compiling commit history with a high ratio and running program analysis techniques that depend on the availability of byte-code.

\section{TARGETING A MODULE AND STUDYING ITS EVOLUTION}

In this section, we explain our approach 1) to detect distinct revisions of a module and ancestry relationships between them, 2) to reach the maximum compilation over commit history and identify all uncompilable revisions, and 3) to achieve scale.

\subsection{Distinct Revisions of a Module and Ancestry Relationships Between Them}

Distinct Revisions of a Module. We target a module to analyze its evolution. The "target module" can be as small as an extension of a library, or as large as a complete software system hosted by a repository. Commit-Impact Analysis [3] is designed to detect "impactful" commits which change the source code in the target 
module, and to calculate their impact by comparing the quality metrics before and after each commit. An impactful can be 1) an "orphan", that introduces the module for the first time, 2) a "simple", that changes its parent's revision of the module, or 3) a "merge", that carries merge agent's combination of the developments on the module over at least two branches leading to it. Figure 1 depicts the evolution of a software system. Node 3 is an impactful orphan. Nodes $6 \mathrm{~b}, 8 \mathrm{a}, 8 \mathrm{~b}$ are impactful simples. Node 9 is an impactful merge.

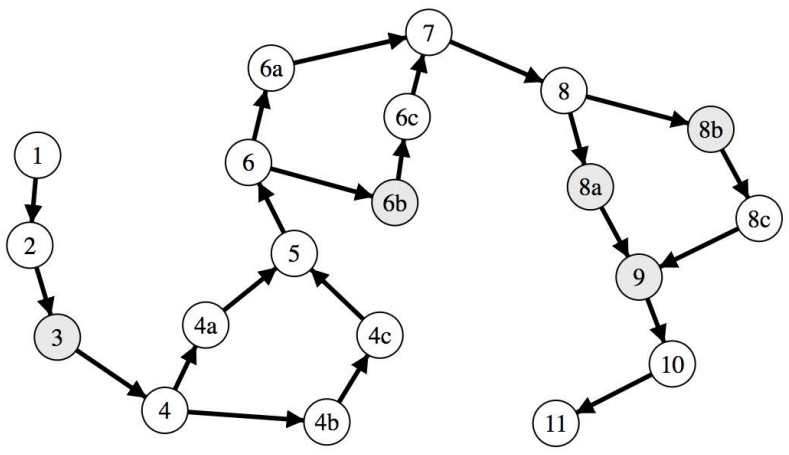

node: commit edge: parent $\rightarrow$ child gray: impactful

Figure 1: A DAG depicting the evolution of a system [3].

The impact of an orphan is the absolute value of quality metrics in the new revision. The impact of a simple is the difference between quality metrics before and after it. The impact of a merge is not calculable by comparing two revisions as it carries the combination of multiple impactfuls over at least two branches [3].

Ancestry Relationships. In order to calculate the impact of an impactful simple node ${ }_{i}$, we need to compare two revisions:

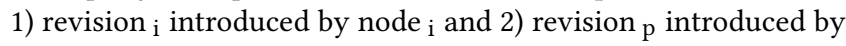
node $_{\mathrm{i}}$ 's direct parent node $\mathrm{p}_{\mathrm{p}}$. Revision $\mathrm{i}_{\mathrm{i}}$ is different from all previous revisions since node ${ }_{\mathrm{i}}$ is an impactful. If node $\mathrm{p}$ is also an impactful then so is revision $\mathrm{p}$. Consequently, we need to analyze both revision $_{\mathrm{i}}$ and revision $\mathrm{p}$ to calculate the impact of node $\mathrm{i}_{\mathrm{i}}$. If node $_{p}$ is unimpactful then revision ${ }_{p}$ is identical to revision ${ }_{a}$ introduced by an ancestor impactful node ${ }_{\mathrm{a}}$. In this case, we can analyze revision $_{\mathrm{a}}$ instead of revision $\mathrm{p}$ to avoid redundancy. We call node ${ }_{a}$, the "impact-parent" of node ${ }_{i}$. Consequently, node ${ }_{i}$ is an "impact-child" of node a.

In order to find node $a$, we generate a Directed Acyclic Graph (DAG) of the evolution. Each node represents a commit. We start the search by looking at node $\mathrm{i}_{\mathrm{i}}$ 's (direct) parent node $\mathrm{p}$. If node $\mathrm{p}$ is an impactful, then we have found the impact-parent. If node $\mathrm{p}$ is unimpactful, there are two possibilities: 1) node $p$ is an unimpactful simple. In this case, we continue the search from node $\mathrm{p}$ 's parent. 2) node $\mathrm{p}$ is an unimpactful merge. In this case, there are two possibilities: a) the revision node $\mathrm{p}_{\mathrm{p}}$ introduces is identical with the revisions all its parents introduce. This indicates that there has been no change on the module over the branches leading to node $\mathrm{p}$. We randomly pick one of the parents and continue the search from it. b) the revision node $\mathrm{p}$ introduces is identical with the revision introduced by one parent and is different from the revisions introduced by other parent(s). This indicates that the merge carries changes introduced in the branch leading to that parent. We pick that parent and continue the search.
We continue this process until we find an impactful to set it as the impact-parent. If we reach an unimpactful orphan without finding the impact-parent, then we treat node $\mathrm{i}_{\mathrm{i}}$ as an impactful orphan. For example, in Figure 1, to find the impact-parent of node $8 \mathrm{~b}$, we look at its parent, node ${ }_{8}$. Node ${ }_{8}$ is a unimpactful simple. We continue the search from node 8 's parent, node 7 . Node 7 is an unimpactful merge. The target module in node 7 is identical to node $6 \mathrm{c}$ as there is an impactful on the branch leading to node $6 \mathrm{c}$. We choose node $6 \mathrm{c}$ and continue the search. Node $6 \mathrm{c}$ is an unimpactful simple. We continue the search from node $6 \mathrm{c}$ 's parent, node ${ }_{6 \mathrm{~b}}$. Node $6 \mathrm{~b}$ is an impactful. We set node $6 \mathrm{~b}$ as the impact-parent of node $8 \mathrm{~b}$ $\left(\right.$ node $_{6 \mathrm{~b}} \rightarrow$ node $_{8 \mathrm{~b}}$ ). Other impact-parent relationships in Figure 1 are $\left(\right.$ node $_{3} \rightarrow$ node $_{6 \mathrm{~b}}$ ) and (node $6 \mathrm{~b} \rightarrow$ node $\left._{8 \mathrm{a}}\right)$.

\subsection{Maximum Compilation}

Reaching the maximum compilation over commit history is necessary when a program analysis technique that depends on byte-code is employed. Uncompilability in post-development analysis can be the result of a developer's fault during development (e.g., syntax error) or compilation environment issues (e.g., missing dependency) during mining. Our goal is to fix the environment issues and identify commits that break the compilation by a developer's fault.

We use the following terminology to categorize impactfuls based on their impact on compilability. An impactful is a "broken" if it produces an uncompilable revision; otherwise, it is a "solid". A broken is a "breaker" if it breaks the compilability of its solid impact-parent; otherwise it is a "carrier". A solid is a "fixer" if it fixes its broken impact-parent; otherwise it is a "neutral". We do not categorize impactful orphans and merges into breaker, carrier, fixer, and neutral as their impact is not identifiable by comparing the compilability of two revisions.

For each system, we modify the default build command to compile the target module and to skip running tests and modifiers. For example, this command for the core module of Google Error-Prune is "mvn clean compile -DskipTests -pl core -am". We compile older revisions using different versions of Java and build tools.

After the first attempt to compile all revisions, we generate a report showing sequences of brokens. Table 1 shows this report for three sequences in a subject system. Each row represents a broken, its impact-parent, and its impact-child. All commits belonging to a sequence are denoted in the same color. All broken impact-parent and impact-child commits are denoted in orange.

Table 1: Report on broken sequences.

\begin{tabular}{|c|c|c|c|c|c|c|c|c|c|c|c|}
\hline \multicolumn{2}{|c|}{ Time } & \multicolumn{2}{|c|}{ Message } & \multicolumn{3}{|c|}{ Commit } & \multicolumn{2}{|c|}{ Author } & \multicolumn{3}{|c|}{ Sequence } \\
\hline Broken & Child & Broken & Child & Broken & Parent & Child & Broken & Child & $\mathbf{L}$ & D & A \\
\hline $6 / 12 / 06$ 13:51 & $6 / 12 / 06$ 13:52 & NUTCH- & NUTCH & $13918 \mathrm{f} 7$ & 1 a47936 & 0c08989 & $\operatorname{dev} 3$ & $\operatorname{dev} 3$ & 1 & 1 & 1 \\
\hline $12 / 29 / 05$ 7:28 & $12 / 29 / 0523: 55$ & A frame & Fix thi & b117756 & b8bd3fi & c4fd3de & dev_2 & dev_2 & 1 & 987 & 1 \\
\hline $\begin{array}{l}5 / 4 / 0512: 38 \\
\end{array}$ & $\begin{array}{l}5 / 4 / 0512: 57 \\
\end{array}$ & Fixed b & Add res & $\begin{array}{l}\mathrm{e} 027 \mathrm{c} 5 \\
\end{array}$ & $4 \mathrm{ffb} 78 \mathrm{~b}$ & a17464e & $\begin{array}{l}\text { dev_1 } \\
\text { deves }\end{array}$ & $\begin{array}{l}\text { dev } 11 \\
\text { dent }\end{array}$ & 6 & 1372 & 2 \\
\hline $5 / 4 / 056: 53$ & $5 / 4 / 0512: 38$ & Whitesp & Fixed b & $4 \mathrm{ffb} 78 \mathrm{~b}$ & $9707 \mathrm{c} 44$ & $10027 c 5$ & $\begin{array}{l}\operatorname{dev} 2 \\
\operatorname{den}\end{array}$ & $\begin{array}{l}\text { dev } 1 \\
\text { des }\end{array}$ & & & \\
\hline 5/4/05 3:10 & 5/4/05 6:53 & Add uti & Whitesp & $9707 c 44$ & $\mathrm{e} 21843 \mathrm{f}$ & $4 f f b 78 b$ & $\begin{array}{l}\text { dev_2 } 2 \\
\text { dent }\end{array}$ & $\begin{array}{l}\text { dev_2 } 2 \\
\text { den }\end{array}$ & & & \\
\hline $5 / 3 / 0515: 32$ & 5/4/05 3:10 & Better & Add uti & e21843f & 6a9871b & $9707 \mathrm{c} 44$ & dev_1 & dev_2 2 & & & \\
\hline $5 / 3 / 05 \quad 14: 07$ & $5 / 3 / 05$ 15:32 & Update & Better & $6 \mathrm{a} 9871 \mathrm{~b}$ & $45 \mathrm{~b} 5 \mathrm{cf} 7$ & e21843f & dev 1 & dev 1 & & & \\
\hline 5/3/05 14:05 & 5/3/05 14:07 & Rewrite & Update & $45 \mathrm{~b} 5 \mathrm{cf} 7$ & aa1b8c5 & $6 \mathrm{a} 9871 \mathrm{~b}$ & dev_1 & dev_1 & & & \\
\hline
\end{tabular}

We investigate the report to find the longest sequences. Exceptionally long sequences are usually the result of a change in the structure and/or the build command, or a missing dependency. For each sequence, we compare the first broken (breaker) with its 
impact-parent and the last broken with its impact-child (fixer) to determine the reason for introduction and resolution of the error.

If the structure has changed, we consider an alternative for the target module. For example, the core module of Apache HttpClient is changed from "httpclient/src/main/java" to "httpclient $5 / \mathrm{src} / \mathrm{main}$ /java". This usually results in an update to the build command. If there is a missing dependency, we fix it. For example, Google MOE has a missing dependency, "joda-time-2.3.jar", over a period. We download it to "lib/" directory. In another example, the SNAPSHOT version of a dependency in Google-Truth is missing. We modify core/pom.xml to replace the SNAPSHOT with a stable version.

After fixing the environment issues, we compile the remaining brokens. We repeat this process until we reach the maximum compilation and identify all brokens caused by a developer's fault. In order to reduce the manual effort involved in this process and to achieve replication and scale, we have designed and implemented an automated solution to compile all revisions using different versions of Java and build tools, to resolve missing dependencies for multiple revisions, and to consider changes in structure and build commands. We explain our solution in Section 3.3.

\subsection{Scalable Analysis}

Compiling and analyzing commit history to assess software quality is computationally expensive. For example, Tufano et al. [21] sequentially analyze the commit history of 200 systems. They only analyze the changed files using a light-weight static analysis technique, still the analysis takes several weeks. Trautsch et al. [19] address the problem of scalability by distributing the analysis of the changed files on HPC clusters. Our approach to achieve scale is distributing the analysis of distinct revisions of a target module over the cloud which allows us to customize the analysis environment and does not require an expensive on-premise infrastructure.

We have designed and implemented SQUAAD [4], a comprehensive cloud-based framework to conduct large-scale empirical studies on software quality evolution. SQUAAD targets a module and identifies its distinct revisions and ancestry relationships between them. It distributes the revisions over the cloud to be compiled and analyzed using static/dynamic techniques. It collects quality metrics calculated by different program analysis tools in a unified relational schema which facilitates multi-perspective analysis. In this paper, we explain SQUAAD's architecture as is depicted in Figure 2.

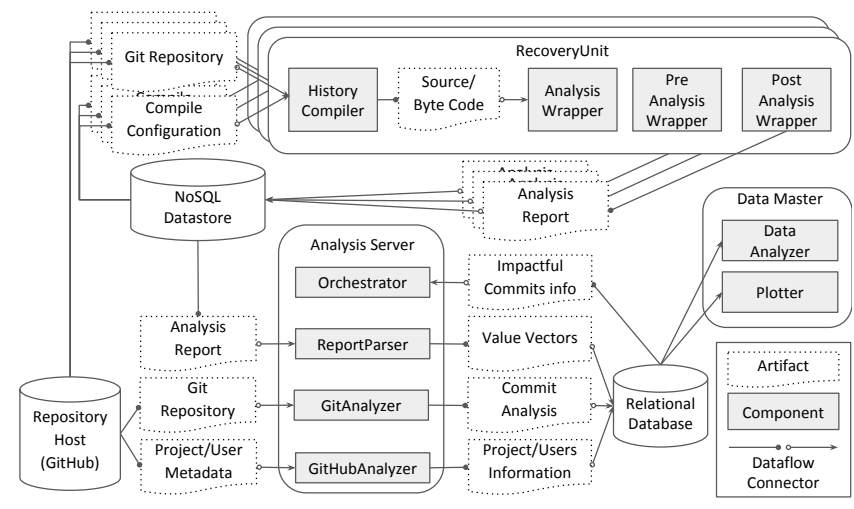

Figure 2: Architecture of SQUAAD.
SQUAAD distributes the analysis over "RecoveryUnit"s. A RecoveryUnit can be a cloud instance, a virtual machine (deployed on an on-premise server), or a process. Each RecoveryUnit downloads the source code of multiple revisions, compiles the source code, and runs program analysis on source/byte code. For complex analyses that need either a server deployment or a specific environment to run, SQUAAD provisions the RecoveryUnits over the cloud.

Each RecoveryUnit contains a "HistoryCompiler" which implements our method to reach the maximum compilation discussed in Section 3.2. The HistoryCompiler receives a "Compile Configuration" file for each subject system that contains its Git repository's address, target module location(s), and instructions(s) to compile the software and fix environment issues. If there are multiple build commands, the HistoryCompiler executes them based on their declaration order. It is capable of compiling older revisions using different versions of Java and build tools.

SQUAAD supports incorporating program analysis tools by providing a standard "AnalysisWrapper" that serves as an interface. Each AnalysisWrapper is accompanied by a "PreAnalysisWrapper" and a "PostAnalysisWrapper". PreAnalysisWrapper prepares environment and data for running the analysis. An AnalysisWrapper is invoked if and only if its PreAnalysisWrapper executes successfully. Some tools require extra work after the analysis is done (e.g., retrieving data from their internal database). This is done by a PostAnalysisWrapper which then stores artifacts generated by the analysis into a "NoSQL Datastore".

We developed "ReportParser"s to retrieve quality metrics from the artifacts generated by program analysis tools. A ReportParser receives a report in a standard format (e.g., JSON) and transforms it to a map of metric-values. This map as well as the revision ID will be stored in a relational database.

SQUAAD distributes the analysis using its "Orchestrator". The Orchestrator interacts with cloud infrastructures to perform cloud management operations, such as launching/stopping/terminating instances and setting up required software. The Orchestrator receives a list of revisions, chronologically sorts them, and uses a round-robin algorithm to schedule the analysis of each revision. This results in a relatively balanced distribution of revisions over RecoveryUnits and reduces the overall mining time.

The "GitAnalyzer" downloads a system's Git repository. It runs a light-weight mining process to retrieve all commits' metadata and stores them in the database. It implements our algorithms to detect impactfuls and their relationships as explained earlier in Section 3.1. This information is later used by the Orchestrator to distribute the revisions. The "GitHubAnalyzer" retrieves organizations/projects' information via GitHub API and stores it in our database. This includes but not limited to, the list of repositories and developers of an organization, as well as the number of forks of a repository. The "DataAnalyzer" and the "Plotter" employ the data generated by other components, run data analytics, and visualize software quality evolution.

\section{EMPIRICAL EVALUATION}

In order to demonstrate the efficiency of our approach to achieve a high compilation ratio and enable a more complete analysis over commit history, we design a large-scale multiple perspective empirical study to answer four research questions. 
RQ1. How efficient is our approach in reaching the maximum compilation and identifying uncompilable commits? The main goal of our approach is to achieve a high compilation ratio over commit history. In this research question, we evaluate to what extent our approach is capable of compiling the history and identifying the impact of each commit on compilability.

RQ2. What are the characteristics of sequences of uncompilable commits? Uncompilability results in an incomplete analysis. In this research question, we investigate the periods where the software is uncompilable to understand how long it lasts and how many impactful commits and developers are involved.

RQ3. Is it feasible to predict uncompilability based on commit metadata? Compilation is computationally expensive. In this research question, we assess the feasibility of predicting whether a commit is uncompilable using commit metadata (i.e., time, message, author) and without considering code differences.

RQ4. How efficient is our approach in identifying change in quality metrics? Reaching the maximum compilation results in a more complete analysis as it minimizes missing data and enables identifying all changes to quality metrics. In this research question, we analyze the compiled revisions to investigate whether we can identify statistically significant difference between two groups of developers in the same organization in terms of changing quality metrics, specially for the metrics with low change frequency.

\subsection{Data Collection}

We analyze the development history of 68 open-source software systems across 3 different organizations (Apache, Google, and Netflix). This dataset is built on the work of [3] including 38 Apache systems, and extends it to include revisions committed in 2017/18 of those 38 Apache systems, as well as 30 new open-source systems from Google and Netflix comprising a total of 37838 distinct revisions and more than 1.5 billion lines of code.

For selecting new subject systems, we retrieve all Java systems owned by Google and Netflix on GitHub using the GitHubAnalyzer. We select a system only if it 1) requires Ant, Maven, or Gradle for compilation and is not an Android, a Bazel, or an Eclipse project, 2) does not require manual installation of other tools (e.g., Protoc) for compilation, 3) is an official product of the organization, and 4) has a core module containing a substantial amount of code. We employ the GitAnalyzer to target the core module and to identify impactfuls in each system and exclude the ones with less than 100 distinct revisions. Table 2 shows the scale of our study.

Table 2: Experiment's scale.

\begin{tabular}{|c|c|c|c|c|c|c|c|}
\hline \multirow{2}{*}{ Org. } & \multirow{2}{*}{ Timespan } & \multirow{2}{*}{ Sys. } & \multicolumn{2}{|c|}{ Developers } & \multicolumn{2}{|c|}{ Commits } & \multirow{2}{*}{$\begin{array}{c}\text { MS } \\
\text { LOC }\end{array}$} \\
\hline & & & All. & Imp. & All & Imp. & \\
\hline Apache & $01 / 02-02 / 18$ & 38 & 1424 & 937 & 46952 & 22627 & 734.3 \\
\hline Google & 08/08-01/18 & 18 & 1217 & 771 & 19249 & 11527 & 760 \\
\hline Netflix & $05 / 11-01 / 18$ & 12 & 641 & 290 & 11379 & 3684 & 36.8 \\
\hline Total & $01 / 02-02 / 18$ & 68 & 3282 & 1998 & 77580 & 37838 & 1531.1 \\
\hline
\end{tabular}

Next, we analyze each system to reach its maximum compilation using the method explained in Section 3.2. We inspect all brokens to confirm that the software is uncompilable as a result of a developer's fault. We also identify all breakers, carriers, fixers, and neutrals.
We analyze all compiled distinct revisions using FindBugs [2] and SonarQube [6]. FindBugs depends on the availability of bytecode. Executing FindBugs is straightforward. It is a one-line bash script command and needs the path to the source/binary files. It also generates a report which can be parsed by a ReportParser. As a result, the challenging part is the compilation. SonarQube does not require byte-code. However, it has its own analysis server and stores the results in an internal database. It requires generating a configuration file for each revision.

To run SonarQube, we develop a PreAnalysisWrapper to deploy SonarQube server on each RecoveryUnit and to wait till the server is available. To analyze each revision, we develop an AnalysisWrapper to generate the SonarQube configuration file and to execute SonarQube on each revision. When the execution of an analysis is done, we need to fetch the data using SonarQube RESTful API. We face four technical challenges in the process of execution and retrieving data from SonarQube server. 1) The server randomly crashes when concurrently analyzing more than three revisions. 2) The result of the analysis is not available through the API immediately after the execution. 3) There is a limitation on the number of data points returned by each request. 4) The server crashes after analyzing a few hundred revisions in a RecoveryUnit.

We resolve the first challenge by distributing the revisions over multiple RecoveryUnits as opposed to concurrently analyzing multiple revisions on a single RecoveryUnit. We resolve the next three challenges by developing a PostAnalysisWrapper that a) periodically checks if the new result is available, b) sends multiple requests to the server and collects each response, c) merges all responses and creates a final report for the analyzed revision, and d) removes the result from SonarQube server to prevent a crash.

We develop two ReportParsers to retrieve quality metrics from the reports generated by FindBugs and SonarQube and to store them in our relational database. The quality metrics used in this study are Lines of Code (LC), Code compleXity (CX), code SMells (SM) and security VuLnerabilities (VL) detected by SonarQube, and Malicious-Code and security problems (MC) detected by FindBugs.

\subsection{Results}

4.2.1 RQ1. How efficient is our approach in reaching the maximum compilation and identifying uncompilable commits? We achieve an average system compilation ratio of $98.4 \%$ for Apache, $98.1 \%$ for Google, and $\overline{93.9 \%}$ for Netflix. Figure 3 shows the distribution of compilation ratio across systems in each organization. The compilation ratio across all revisions in each organization is $98.4 \%$ for Apache, $99.0 \%$ for Google, and $94.3 \%$ for Netflix.

We identify 348 breakers, 312 carriers, 347 fixers, and 36,141 neutrals. The number of breakers and fixers can be different as each breaker can have 1) no fixer if it is never fixed, 2) one fixer, or 2) more than one fixer if it is fixed in more than one branch starting from it. Table 3 shows the number of commits in each category.

In $47 \%$ of systems, we reach the maximum compilation using one instruction (i.e., the default build command). Also in $30 \%$ of them, one alternative suffices to fix environment issues (i.e., fixing a missing dependency and addressing a change in structure/build tool). However, up to 5 different instructions are required for some systems. Table 4 shows the frequency of the number of required 


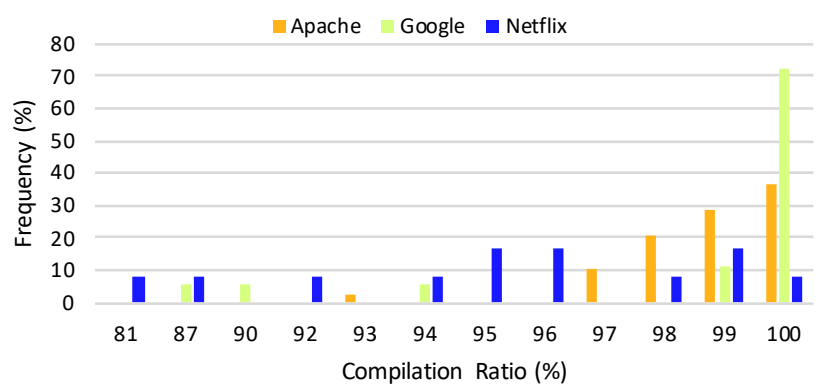

Figure 3: Compilation ratio distribution.

Table 3: Number of breakers, carriers, fixers, and neutrals.

\begin{tabular}{lccccccc}
\hline & \multicolumn{3}{c}{ Broken } & & \multicolumn{3}{c}{ Solid } \\
\cline { 2 - 4 } \cline { 7 - 8 } Org. & Breaker & Carrier & Total & & Fixer & Neutral & Total \\
\hline Apache & 215 & 139 & 354 & & 220 & 21776 & 21996 \\
Google & 44 & 69 & 113 & & 46 & 11244 & 11290 \\
Netflix & 89 & 104 & 193 & & 81 & 3121 & 3202 \\
\hline Total & 348 & 312 & 660 & & 347 & 36141 & 36488 \\
\hline
\end{tabular}

instructions and build tools for systems in each organization. For example, 2 Netflix systems use both Maven and Gradle over their commit history and 13 Apache systems need two instructions.

The result of this analysis shows that our approach is capable of achieving a high compilation ratio and identifying the impact of commits on compilability. It also shows that our approach does not demand an extensive number of instructions to reach the maximum compilation as only 5 systems need more than three instructions.

Table 4: Frequency of the number of instructions and tools.

\begin{tabular}{|c|c|c|c|c|c|c|c|c|c|c|}
\hline \multirow[b]{2}{*}{ Org. } & \multicolumn{5}{|c|}{ Instructions } & \multicolumn{5}{|c|}{ Build Tools } \\
\hline & 1 & 2 & 3 & 4 & 5 & A & $\mathbf{M}$ & G & AM & MG \\
\hline Apache & 18 & 13 & 4 & 2 & 1 & 2 & 33 & 0 & 3 & 0 \\
\hline Google & 7 & 6 & 4 & 1 & 0 & 2 & 12 & 1 & 2 & 1 \\
\hline Netflix & 7 & 1 & 3 & 0 & 1 & 0 & 0 & 10 & 0 & 2 \\
\hline Total & 32 & 20 & 11 & 3 & 2 & 4 & 45 & 11 & 5 & 3 \\
\hline
\end{tabular}

4.2.2 RQ2. What are the characteristics of sequences of uncompilable commits? We define a "broken sequence" as a series of back-to-back impactfuls starting with a breaker and ending with a fixer. We identify a total of 303 broken sequences (210 for Apache, 43 for Google, and 50 for Netflix) in our dataset. For each sequence, the "length" is the number of brokens, the "duration" is the time between the breaker and the fixer, and the "involvement" is the number of authors. Figure 4 shows the distribution of these three characteristics. For example in Netflix, $74 \%$ of broken sequences contain one commit, $33 \%$ last between one minute to an hour, and $89 \%$ involve one author.

In $79 \%$ of sequences, length is 1 meaning that the breaker is the impact-parent of the fixer. However, in $4 \%$ of sequences, more than 10 brokens are involved which can imply that the code is repetitively changing without it even being compilable. In $57 \%$ of
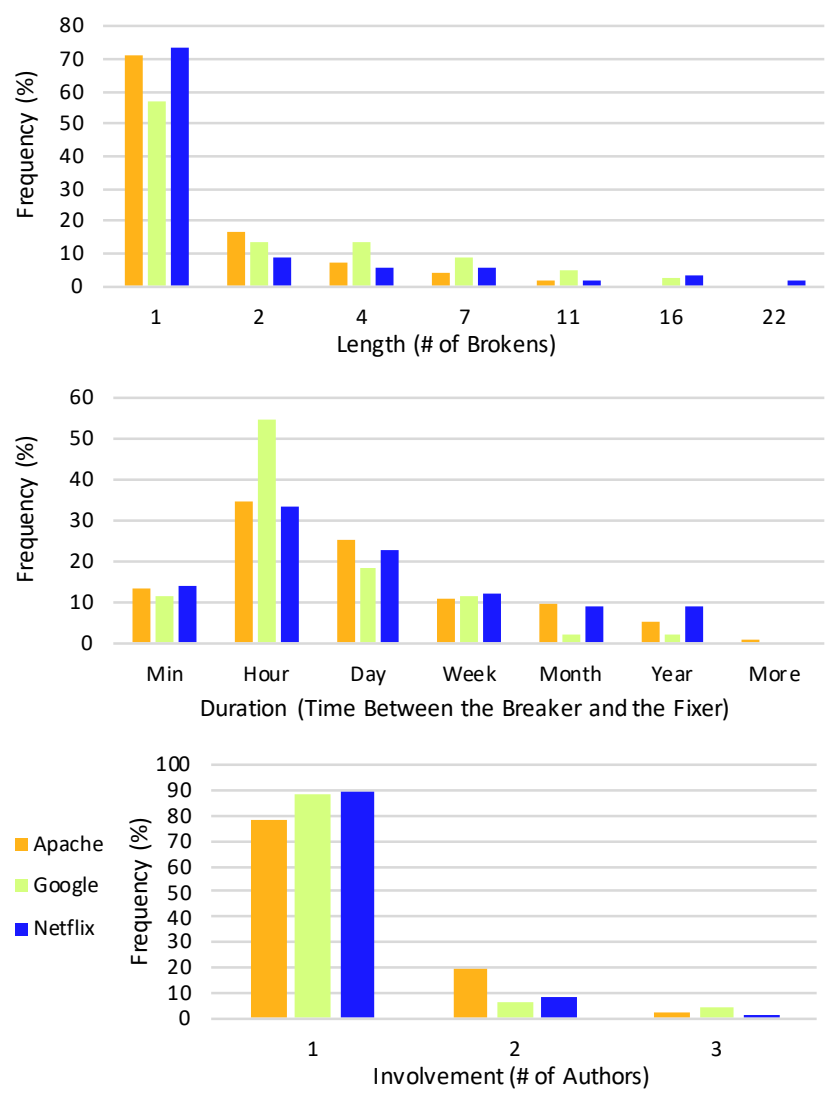

Figure 4: Distribution of characteristics of sequences.

sequences, duration is not more an hour. Nevertheless, in $17 \%$ of sequences the software remains uncompilable for several weeks or even months. Although, in $93 \%$ of sequences involvement is 1 ; we identify 56 sequences that 2 authors and 8 sequences that 3 authors are changing the software while it is uncompilable.

4.2.3 RQ3. Is it feasible to predict uncompilability based on commit metadata? Next, we study the difference between the metadata (i.e., time, message, author) of breaker, carrier, fixer, neutral to assess the feasibility of creating a model to predict uncompilability.

Time. For each impactful, we define the "interval" as the time difference between the commit and its impact-parent. Although it takes several minutes to build most of our subject systems, we observe that a large number of impactfuls have a relatively short interval. For example, we identify 3,056 impactfuls with an interval less than a minute. This can indicate that the developer had not attempted to build the software before committing.

To further investigate, we calculate the interval for all breakers, carriers, fixers, and neutrals. Logically, the intervals should be positive numbers since every commit is younger than its parent, but we identify 1,453 impactfuls with a negative interval. The negative values can be a result of inaccurate time on the local machine of the developer, or a post-commit modification in the metadata. We exclude the impactfuls with negative intervals and calculate the Cumulative Distribution Function (CFD) for $\log _{7.75 s}$ (interval). We 
use $7.75 \mathrm{~s}$ as the scale to present the result in a more understandable way as $\log _{7.75 s}(1 \mathrm{~m})=2$ and $\log _{7.75 s}(1 \mathrm{~h})=4$. Figure 5 depicts the CDFs. For example, it shows that almost $30 \%$ of breakers and neutrals in Google happen in less than an hour. However, this ratio is almost $70 \%$ for carrier and fixer.
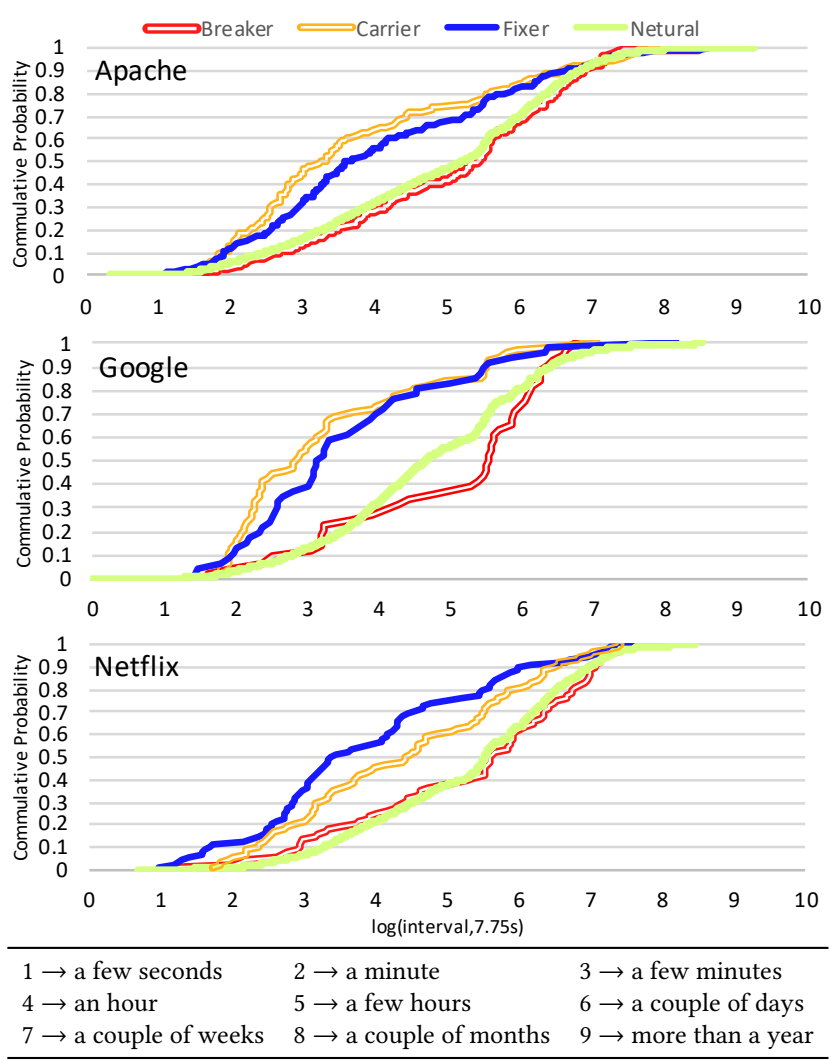

Figure 5: CDF of breaker, carrier, fixer, and neutral interval.

Interestingly, CFDs show no difference between breaker and neutral in short intervals (i.e., less than an hour). This contradicts our initial assumption and previous observation [3] that when developers commit too early (apparently without attempting the compilation), the chance of introducing an error is higher. However, carrier and fixer tend to have shorter intervals in comparison with breaker and neutral. In all three organizations, a high ratio of carriers and fixers are committed in less than a few minutes. This can imply that when a developer introduces a compile error, oftentimes they either fix it quickly or continue the development by committing more changes in a short period without fixing the existing errors. In addition, it shows that carrier and fixer do not happen very late and their distribution is not that wide, while the distribution of breaker and neutral is wide. This can imply that developers do not usually commit a broken and leave the software for a long interval.

Motivated by these observations, we investigate whether we could use an impactful's interval to create a model to predict uncompilability in its impact-parent. We convert the interval to $\log _{7.75 \mathrm{~s}}$ scale and consider the value of zero where the interval is negative. We calculate the probability distribution of interval of impactfuls where their impact-parent is broken and solid. The result as depicted in Figure 6 shows the difference between the two distributions.

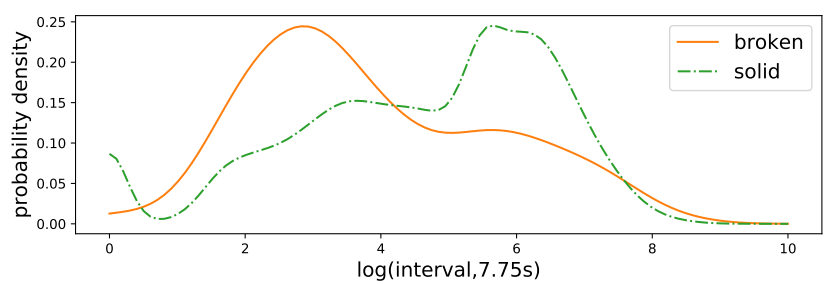

Figure 6: Probability density of interval in $\log _{7.75 \mathrm{~s}}$ scale.

Message. In manual inspection, we observe that in many broken sequences, there are subsequent commits with very similar or almost identical messages. To quantify this observation, we implement string matching techniques suggested by Wael et al. [8] to determine the similarity of the messages of impactfuls and their impact-parent. Most messages are short and ungrammatical and some contain symbolic words (e.g., URL). We find that many similar messages are, in fact, almost identical rather than being a paraphrase of one another. Therefore, instead of assessing semantics similarity, we implement Jaccard Distance to compare the two messages. The result, as depicted in Figure 7, shows that the probability of message similarity of an impactful with its impact-parent where the parent is broken is different from those where the parent is solid. It can be seen that the probability mass of broken is distributed to the right of the $\mathrm{x}$-axis more than that of solid. This can be explained by that a high similarity between messages indicates that the first commit is incomplete and needs follow up commits to complete the same task.

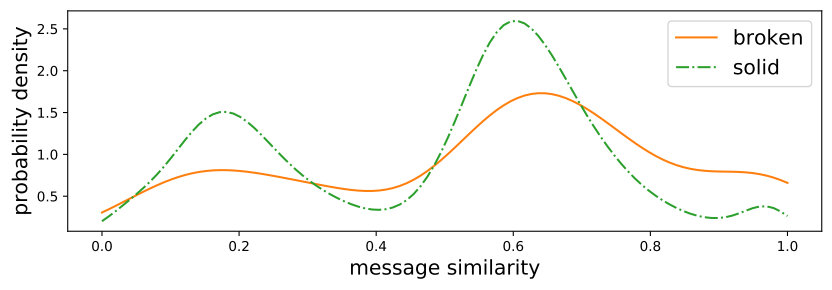

Figure 7: Probability density of message similarity.

In addition, we observe that the messages of many back-to-back brokens mention the same issue number. To quantify this observation, we extract the issue numbers from the message of impactfuls and their impact-parent. The result shows that $55 \%$ of commits whose parent is broken mention the same issue number as the parent. This ratio is $30 \%$ when the parent is solid.

We observe that developers even acknowledge the existence of the error when fixing it by saying specific words (e.g., "oops" and "sorry") in the message. We analyze the message of impactfuls to identify the words that could be used as an indicator of existence of compile error in their impact-parent using Naive Bayes and Logistic Regression. We analyze the parameters of the model, which are probability estimation in Naive Bayes, and coefficients of decision boundary in Logistic Regression. The result shows that words "oops", "compile", "compilation", "missing", "missed", "adapted" and 
phrases that contain an issue number (e.g., "drill-4134", "opennlp580 ") contribute to the prediction model.

Author. The authors of impactfuls and their impact-parent are the same in $62 \%$ of breakers, $67 \%$ of carriers, $88 \%$ of fixers, and $63 \%$ of neutrals. However, our analysis shows that considering this as a feature does not contribute to the prediction model.

In summary, the following 6 features from a pair of an impactful and its impact-parent are extracted for training a model to predict uncompilability in the parent: 1) The interval of the commit, 2) whether it is less than an hour, and 3) whether it is negative. 4) The similarity between messages of the commit and its parent, 5) whether they mention the same issue number, and 6) whether the message contains some specific words (e.g., "oops").

Our dataset is composed of 37,148 pairs of impactfuls and their impact-parent, and is divided to 36,489 pairs with a solid parent (i.e., breaker or neutral), and 659 pairs with a broken parent (i.e., carrier or fixer). Since the dataset is imbalanced, we cannot use it to train the model directly. He et al. [11] suggest techniques for dealing with imbalanced data. We implement the random undersampling technique, which randomly selects a set of majority class instances, and removes these instances from that class to make the remaining data balanced. The balanced dataset contains 1,200 pairs. We randomly split it to a training dataset composed of 900 pairs and a test dataset composed of 300 pairs. We train the logistic regression model, test it, and achieve an F1-score of 0.89 and an AUC of 0.96. Table 5 shows the confusion matrix. This model shows the feasibility of applying statistical learning to predict uncompilability based on commit metadata and without considering code differences.

Table 5: Confusion matrix.

\begin{tabular}{lccc}
\hline $\mathrm{n}=300$ & Predicted Solid & Predicted Broken & Total \\
\hline Actual Solid & 153 & 9 & 162 \\
Actual Broken & 19 & 119 & 138 \\
\hline Total & 172 & 128 & \\
\hline
\end{tabular}

4.2.4 RQ4. How efficient is our approach in identifying change in quality metrics? Some quality metrics (e.g., security) have a low change frequency ratio (i.e. less than a few percent) over commit history [3]. Consequently, a statistical analysis on their change 1) can significantly be affected by missing data and 2) requires a large quantity of data. We minimize missing data (by reaching the maximum compilation) and collect quality metrics from a large number of commits. To empirically evaluate this, we design an experiment to divide the developers of each organization into two groups and ask whether there is a difference between them in terms of missing data and change frequency in quality metrics.

We use the affiliation status of developers to divide them into two groups. We assume a developer to be "affiliated" if they use an official email of an organization while committing to a repository owned by that organization; otherwise, they are "external". The official email domain for Apache is @apache.org, for Google is @google.com, and for Netflix is @netflix.com.

We calculate the ratio of brokens to understand the extent of missing data in our dataset for affiliated and external of each organization. We also calculate change frequency ratio in each metric by comparing the metric value in neutrals and their parent. In order to
Table 6: Ratio of change to quality metrics.

\begin{tabular}{|c|c|c|c|c|c|c|c|c|}
\hline \multirow{2}{*}{\multicolumn{2}{|c|}{ Affiliation }} & \multirow{2}{*}{$\begin{array}{c}\text { broken } \\
(\%)\end{array}$} & \multirow{2}{*}{$\begin{array}{c}\text { neutral } \\
(\#)\end{array}$} & \multicolumn{5}{|c|}{ Change Ratios (\%) } \\
\hline & & & & LC & $\mathrm{CX}$ & SM & MC & VL \\
\hline \multirow{3}{*}{ Apache } & A & 1.55 & 16810 & 69.80 & 53.39 & 45.42 & 2.77 & 5.97 \\
\hline & E & 1.58 & 4966 & 85.11 & 71.07 & 57.34 & 3.25 & 8.46 \\
\hline & $\boldsymbol{P}$ & 1 & & 0 & 0 & 0 & 0.559 & 0 \\
\hline \multirow{3}{*}{ Google } & A & 0.45 & 8734 & 77.59 & 59.18 & 44.74 & 0.28 & 2.74 \\
\hline & E & 3.09 & 2510 & 77.34 & 61.38 & 53.03 & 2.02 & 6.24 \\
\hline & $P$ & 0 & & 1 & 0.429 & 0 & 0 & 0 \\
\hline \multirow{3}{*}{ Netflix } & A & 7.52 & 1610 & 78.45 & 62.05 & 54.60 & 1.30 & 8.82 \\
\hline & E & 4.42 & 1511 & 77.22 & 60.73 & 49.80 & 1.32 & 8.87 \\
\hline & $\boldsymbol{P}$ & 0.001 & & 0.963 & 0.974 & 0.079 & 1 & 1 \\
\hline & & & ls & & ode & $\begin{array}{l}C X: \\
V L:\end{array}$ & & es \\
\hline
\end{tabular}

compare the ratios, we perform a Games-Howell statistical significance test as there is difference in the sample sizes and variances. We consider a $5 \%$ confidence interval or P-Value less than 0.05. 9 (out of 18) tests identify statistically significant difference. Table 6 shows the ratio of brokens and the number of neutrals by affiliated and external, and the results of tests.

In Apache, there is no statistically significant difference between affiliated and external in broken ratio. However, affiliated has a lower change frequency ratio in LC, CX, SM, and VL. The ratio of $\mathrm{MC}$ is lower in affiliated but the difference is not significant. As opposed to Apache, there is no significant difference between affiliated and external of Google and Netflix in ratio of LC and CX. The broken ratios of Google external and Netflix affiliated are significantly higher. For SM, VL and MC, Google affiliated has significantly lower ratios, but there is no significant difference between affiliated and external of Netflix.

This analysis shows that our approach is capable of minimizing the number of missing data points and collecting a large quantity of data on software quality. It also shows that we can use the collected data to identify statistically significant difference between the impact of two groups of developers within an organization, even for quality metrics with low change frequency ratio.

4.2.5 Replication. The detailed list of our subject systems, the impactfuls and their relationships, the compilability of each commit, and the quality metrics calculated for the compiled revisions are available in our replication package ${ }^{1}$

Note that our approach to collect data does not rely on an expensive on-premise infrastructure (e.g., an HPC cluster or a multiprocessor server machine). The compilation and analysis of all revisions of Google and Netflix systems are done by distributing the analysis over tens of AWS m5.large nodes (2 vCPUs, 8GB Memory). The analysis costs $\$ 102$ and takes a total of 1884 hours. Consequently, its replication using 20 cloud instances should take less than a week.

\section{DISCUSSION}

\subsection{Benefits and Risks of Focusing on a Module}

5.1.1 Benfits. We have identified multiple benefits for focusing on a module while conducting the study presented in this paper.

${ }^{1}$ https://figshare.com/s/7c3d3569932566649047 
It provides a more complete view of evolution. In several instances, we have observed that a commit introduces a compile error in one module and causes the whole software to be uncompilable over a period; however, other modules are compilable and are evolving. For example, in Netflix-Spectator ${ }^{2}$ commit fbeb719, a compile error in a unit test breaks the build. This causes the unavailability of byte-code which results in missing data and an incomplete analysis. After focusing on the core module and skipping tests, we achieve a $100 \%$ compilation ratio and obtained a complete view of the evolution of the core module.

It facilitates manual inspection of individual data points which is a labor intensive task. In Apache CXF-Fediz ${ }^{3}$, commit b06255a changes the core module and uses a new interface, which causes a compile error. The error is fixed in the next impactful (44d7340) which is a small commit that comments out the line containing the error and says "Switching to WSS4J 2.0.0-rc1 + commenting some stuff as a result". There are four other commits changing code in other modules between these two commits that can be skipped when inspecting why the core module is uncompilable.

It reduces the cost and complexity of analysis. Apache Tiles ${ }^{4}$ is a templating framework for user interface development. It is designed to be integrated with a variety of other systems. Its core module is accompanied with multiple smaller components (e.g., sdk, agents, and plugins). Although majority (70\%) of developers change the core module at least once, only $24 \%$ of commits are impactful. Instead of analyzing all commits to understand the evolution of the core module, we can focus on that smaller subset.

It provides a more accurate analysis by omitting irrelevant changes. Apache Santuario ${ }^{5}$ provides implementation of primary security standards for XML. Its core module ("src/main") is accompanied by a large number of tests ("src/test") comprising $30 \%$ of all source files. Consequently, $34 \%$ of commits do not change code in the core module and including them may cause inaccuracy when analyzing the frequency of change to a quality metric.

5.1.2 Risks. Focusing on a module to achieve a high compilation ratio comes at the price of skipping other modules and artifices.

In order to understand to what extent this happens, we calculate the ratio of commits and developers that change code in the core module. Figure 8 shows the distribution of these ratios across systems in each organization. For example, it shows that in $50 \%$ of Netflix systems, between $20 \%$ and $30 \%$ commits, and in $44 \%$ of Google systems, between $70 \%$ and $80 \%$ of developers are impactful.

This shows that focusing on a target module (e.g., the core module in this study), may result in missing a large number of changes. 1) If those modules are irrelevant, then including their changes may degrade the analysis. For example, in a study of architectural stability of a library, introducing a plug-in is not necessarily an architectural change. However, architectural change metrics [5] detect it as a change since new entities are introduced. 2) If they are relevant, they could be analyzed either separately or in combination with other modules. As explained in Section 3.1, a module can be as small as a plug-in or as a large as the whole software. For example,

\footnotetext{
${ }^{2}$ https://github.com/netflix/spectator

${ }^{3}$ https://github.com/apache/cxf-fediz

${ }^{4}$ https://github.com/apache/tiles

${ }^{5}$ https://github.com/apache/santuario-java
}

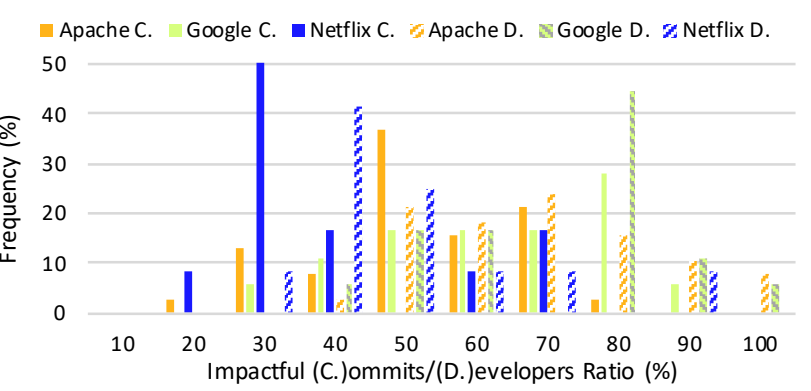

Figure 8: Impactful commits/developers ratio distribution.

in a study of compilability of the whole software, one can target its core module first to fix the compilation environment issues, then gradually include other modules, instead of targeting the whole software and fixing the issues in different modules all together.

\subsection{Threats to Validity}

We discuss the threats to validity of our empirical study based the guidelines by Wieringa et al. [23].

External Validity. The main threats involve our subject systems: 1) We study a limited number of systems. However, we select 68 systems that vary along multiple dimensions, including size, number of commits and developers, owner, used build tools, and domain. 2) We study Java systems. However, our approach to focus on a module is not limited to just Java systems and can be applied to any evolving system hosted in Git. 3) We study open-source systems. Although we do not have access to industry-scale closedsource systems, we select our systems from non-profit (Apache) and for-profit (Google and Netflix) organizations. Another threat is that our algorithm to detect relationships between impactfuls is designed based on Git branching model. Further research should be conducted to investigate if our findings hold for other VCSs.

Conclusion Validity. The main threat involves our manual inspection to confirm uncompilability of commits. We inspect the output generated by build tools to confirm the error for all brokens. The list of brokens is available in our replication package. Another threat involves the statistical analyses used to answer RQ3. We use multiple techniques to measure message similarity and identifying keywords that are an indicator of a compile error.

Internal Validity. Relying on static code analysis tools, which might have false positives and false negatives, to measure quality metrics is an internal threat to validity of our $R Q 4$. However, we use two well-known static analysis tools that are widely-used by open-source community. Another threat involves depending on commit metadata that might be inaccurate/changed. We identify impactfuls that are older than their parent, and consider it as feature in our prediction model.

Construct Validity. The main threat is that we do not study what compilation ratio we could achieve by analyzing the whole software (instead of the core module) and compare it with the results of $R Q 1$. However, in Section 5.1.1, we discuss one of the cases in which we achieve a $100 \%$ compilation ratio although there is a compile error in other artifacts. Also, in Section 2 we discuss the related work that compile the whole software and achieve a low compilation ratio. 


\section{CONCLUSIONS}

We successfully design, develop, and empirically evaluate an approach to achieve a high compilation ratio and enable a more efficient analysis over commit history by focusing on a module instead of the whole software. We design algorithms to target a module and identify its distinct revisions and evolutionary relationships between them. We explain how to reach the maximum compilation using our tool-assisted approach and how to scale the analysis of thousands revisions by distributing them over the cloud. We implement our approach in SQUAAD framework and demonstrate its efficiency by studying the evolution of the core module in 68 industry-scale open-source software systems across Apache, Google, and Netflix. We assess the compilability of all revisions. We analyze the uncompiled sequences to understand how long the software is uncompilable, how many commits and developers are involved, and whether we could predict uncompilability based on commit metadata. We analyze the compiled revisions to study how developers impact software quality.

The most important result from our study is that targeting a module and analyzing its evolution enables achieving a high compilation ratio, provides a more complete and accurate analysis of software quality evolution, reduces the cost and complexity of analysis, and facilitates manual inspection. Our analysis shows that when compilation breaks by a commit, in most cases, the same developers immediately resolves the problem in the next commit. However, there can be long periods over which multiple subsequent commits are uncompilable and multiple developers are actively committing code. It also shows that the metadata of the next commit can be used to create a model for predicting uncompilability, and that there can be a statistically significant difference between affiliated and external developers of an organization on how they change different quality metrics.

Achieving a high compilation ratio over commit history opens up multiple opportunities to extend the analysis presented in this paper for the future work. We plan to study the impact of developers on software architecture using recovery techniques that consider the relationships between files, as well as test-coverage that depends on the availability of byte-code and requires an execution environment. We also plan to extend our fourth research question to analyze the difference between groups of developers within and across organizations not only in terms of the frequency of change, but also an increase and a decrease in quality metrics.

\section{REFERENCES}

[1] C. V. Alexandru, S. Panichella, and H. C. Gall. 2017. Reducing redundancies in multi-revision code analysis. In 2017 IEEE 24th International Conference on Software Analysis, Evolution and Reengineering (SANER). 148-159. https://doi. org/10.1109/SANER.2017.7884617

[2] N. Ayewah, D. Hovemeyer, J. D. Morgenthaler, J. Penix, and W. Pugh. 2008 Using Static Analysis to Find Bugs. IEEE Software 25, 5 (Sept 2008), 22-29. https://doi.org/10.1109/MS.2008.130

[3] P. Behnamghader, R. Alfayez, K. Srisopha, and B. Boehm. 2017. Towards Better Understanding of Software Quality Evolution through Commit-Impact Analysis In 2017 IEEE International Conference on Software Quality, Reliability and Security (QRS). 251-262. https://doi.org/10.1109/QRS.2017.36

[4] P. Behnamghader and B. Boehm. 2018. Towards Better Understanding of Software Maintainability Evolution. In 2018 Conference on Systems Engineering Research (CSER 2018). Charlottesville, USA.

[5] Pooyan Behnamghader, Duc Minh Le, Joshua Garcia, Daniel Link, Arman Shahbazian, and Nenad Medvidovic. 2017. A large-scale study of architectural evolution in open-source software systems. Empirical Software Engineering 22, 3 (01
Jun 2017), 1146-1193. https://doi.org/10.1007/s10664-016-9466-0

[6] G. Ann Campbell and Patroklos P. Papapetrou. 2013. SonarQube in Action (1st ed.). Manning Publications Co., Greenwich, CT, USA.

[7] Robert Dyer, Hoan Anh Nguyen, Hridesh Rajan, and Tien N. Nguyen. 2015. Boa: Ultra-Large-Scale Software Repository and Source-Code Mining. ACM Trans. Softw. Eng. Methodol. 25, 1, Article 7 (Dec. 2015), 34 pages. https://doi.org/10. $1145 / 2803171$

[8] W. H. Gomaa and A. A. Fahmy. 2013. A Survey of Text Similarity Approaches. International fournal of Computer Applications 68, 13 (April 2013), 13-18. https: //doi.org/10.5120/11638-7118

[9] F. Hassan, S. Mostafa, E. S. L. Lam, and X. Wang. 2017. Automatic Building of Java Projects in Software Repositories: A Study on Feasibility and Challenges. In 2017 ACM/IEEE International Symposium on Empirical Software Engineering and Measurement (ESEM). 38-47. https://doi.org/10.1109/ESEM.2017.11

[10] F. Hassan and X. Wang. 2017. Change-Aware Build Prediction Model for Stall Avoidance in Continuous Integration. In 2017 ACM/IEEE International Symposium on Empirical Software Engineering and Measurement (ESEM). 157-162. https: //doi.org/10.1109/ESEM.2017.23

[11] H. He and E. A. Garcia. 2009. Learning from Imbalanced Data. IEEE Transactions on Knowledge and Data Engineering 21, 9 (Sept 2009), 1263-1284. https://doi. org/10.1109/TKDE.2008.239

[12] D. M. Le, P. Behnamghader, J. Garcia, D. Link, A. Shahbazian, and N. Medvidovic. 2015. An Empirical Study of Architectural Change in Open-Source Software Systems. In 2015 IEEE/ACM 12th Working Conference on Mining Software Repositories. 235-245. https://doi.org/10.1109/MSR.2015.29

[13] C. Macho, S. McIntosh, and M. Pinzger. 2018. Automatically repairing dependencyrelated build breakage. In 2018 IEEE 25th International Conference on Software Analysis, Evolution and Reengineering (SANER). 106-117. https://doi.org/10.1109/ SANER.2018.8330201

[14] Y. K. Malaiya, M. N. Li, J. M. Bieman, and R. Karcich. 2002. Software reliability growth with test coverage. IEEE Transactions on Reliability 51, 4 (Dec 2002), 420-426. https://doi.org/10.1109/TR.2002.804489

[15] Hyunmin Seo, Caitlin Sadowski, Sebastian Elbaum, Edward Aftandilian, and Robert Bowdidge. 2014. Programmers' Build Errors: A Case Study (at Google). In Proceedings of the 36th International Conference on Software Engineering (ICSE 2014). ACM, New York, NY, USA, 724-734. https://doi.org/10.1145/2568225. 2568255

[16] Matúš Sulír and Jaroslav Porubän. 2016. A Quantitative Study of Java Software Buildability. In Proceedings of the 7th International Workshop on Evaluation and Usability of Programming Languages and Tools (PLATEAU 2016). ACM, New York, NY, USA, 17-25. https://doi.org/10.1145/3001878.3001882

[17] N. M. Tiwari, G. Upadhyaya, H. A. Nguyen, and H. Rajan. 2017. Candoia: A Platform for Building and Sharing Mining Software Repositories Tools as Apps. In 2017 IEEE/ACM 14th International Conference on Mining Software Repositories (MSR). 53-63. https://doi.org/10.1109/MSR.2017.56

[18] Fabian Trautsch, Steffen Herbold, Philip Makedonski, and Jens Grabowski. 2016. Adressing Problems with External Validity of Repository Mining Studies Through a Smart Data Platform. In Proceedings of the 13th International Conference on Mining Software Repositories (MSR '16). ACM, New York, NY, USA, 97-108. https: //doi.org/10.1145/2901739.2901753

[19] Fabian Trautsch, Steffen Herbold, Philip Makedonski, and Jens Grabowski. 2018. Addressing problems with replicability and validity of repository mining studies through a smart data platform. Empirical Software Engineering 23, 2 (01 Apr 2018), 1036-1083. https://doi.org/10.1007/s10664-017-9537-x

[20] Michele Tufano, Fabio Palomba, Gabriele Bavota, Massimiliano DiÃĆÂăPenta, Rocco Oliveto, Andrea DeÃĆÂăLucia, and Denys Poshyvanyk. [n. d.]. There and back again: Can you compile that snapshot? fournal of Software: Evolution and Process 29, 4 ([n. d.]), e1838. https://doi.org/10.1002/smr.1838 arXiv:https://onlinelibrary.wiley.com/doi/pdf/10.1002/smr.1838 e1838 smr.1838.

[21] M. Tufano, F. Palomba, G. Bavota, R. Oliveto, M. Di Penta, A. De Lucia, and D. Poshyvanyk. 2015. When and Why Your Code Starts to Smell Bad. In 2015 IEEE/ACM 37th IEEE International Conference on Software Engineering, Vol. 1. 403-414. https://doi.org/10.1109/ICSE.2015.59

[22] M. Tufano, F. Palomba, G. Bavota, R. Oliveto, M. D. Penta, A. De Lucia, and D. Poshyvanyk. 2017. When and Why Your Code Starts to Smell Bad (and Whether the Smells Go Away). IEEE Transactions on Software Engineering 43, 11 (Nov 2017), 1063-1088. https://doi.org/10.1109/TSE.2017.2653105

[23] Roelf J. Wieringa. 2014. Design science methodology for information systems and software engineering. Springer. https://doi.org/10.1007/978-3-662-43839-8 10.1007/978-3-662-43839-8. 